\title{
X-Ray Diffraction Studies on Liquids at Very High Pressures along the Melting Curve. I. Methods and Techniques
}

Keith H. Brown

J. Dean Barnett

jdeanbarnett@gmail.com

Follow this and additional works at: https://scholarsarchive.byu.edu/facpub

Part of the Astrophysics and Astronomy Commons, and the Physics Commons

\section{Original Publication Citation}

Brown, Keith H. and Dean J. Barnett."X-Ray Diffraction Studies on Liquids at Very High Pressures along the Melting Curve. I. Methods and Techniques." The Journal of Chemical Physics 57 (1972): 29-215.

\section{BYU ScholarsArchive Citation}

Brown, Keith H. and Barnett, J. Dean, "X-Ray Diffraction Studies on Liquids at Very High Pressures along the Melting Curve. I. Methods and Techniques" (1972). Faculty Publications. 789.

https://scholarsarchive.byu.edu/facpub/789 


\title{
X-Ray Diffraction Studies on Liquids at Very High Pressures along the Melting Curve. I. Methods and Techniques*
}

\author{
Keith H. Brown $\dagger$ and J. Dean Barnett \\ Department of Physics, Brigham Young University, Provo, Utah 84601
}

(Received 2 March 1972)

\begin{abstract}
A technique using the tetrahedral anvil high-pressure $x$-ray system has been developed to extract coherent $x$-ray intensity patterns of liquids near melting from measured scattering curves in the presence of high-background counts. Radial distribution functions for liquids along the melting curve to pressures of at least $50 \mathrm{kbar}$ can be obtained. At each pressure scattering curves are recorded for the liquid and solid on opposite sides of the melting curve and a subtraction is made to eliminate background and incoherent scattering. Theoretical estimates of the thermal diffuse scattering from the solid are added to the appropriately corrected difference patterns to yield the coherent intensity patterns for the liquid. The technique is directed to the measurement of pressure-induced changes rather than absolute measurement of the intensity patterns and the radial distribution functions.
\end{abstract}

\section{INTRODUCTION}

Many investigations of the structure of liquids over a wide range of temperatures at, or near, atmospheric pressure have been made using diffraction techniques. General techniques and procedures have been reviewed elsewhere. ${ }^{1-3}$ Methods for extending these studies in a limited way to high pressures (to $100 \mathrm{kbar}$ ) are presented here.

Development of melting and freezing theories has been hampered by a lack of information about the liquid state at high pressures along the melting curve. An adequate theory describing the liquid-solid transformation should predict the observed melting curves as well as the change due to melting in the thermodynamical variables such as entropy and volume. This lack of information provided the incentive to undertake this study.

Prediction of the change in entropy associated with melting requires information about the structure or ordering in the liquid state. The atomic structure in liquids is generally described by the radial distribution function. The purpose of Paper I of this series is to present a technique for obtaining such information; Paper $\mathrm{II}^{4}$ will discuss the $\mathrm{x}$-ray intensity patterns and the resulting radial distribution functions obtained for liquid sodium at various points along the melting curve.

The radial distribution function or RDF is obtained from the coherent scattering intensity pattern of the liquid. In order to obtain a meaningful coherent intensity pattern, one must correct the observed intensity pattern for polarization, absorption, background scattering, fluorescence, and incoherent scattering. Limitations associated with the production of very high pressures and simultaneous high temperatures complicate these corrections. In liquid studies it is necessary, but often difficult, to separate the diffraction pattern due to the container from the intensity pattern of the liquid. At atmospheric pressure appropriate experimental procedures can often minimize or eliminate any scattering from the container. Containment of the liquid at the extreme pressures involved in this study requires the use of a pressure-transmitting medium and cell of considerable size and strength. This "container" adds significantly to the background intensity by diffracting the $x$ rays and also complicates the absorption correction due to changing geometry and density. Absorption in the pressure-transmitting medium and cell restricts one to $\mathrm{MoK \alpha}$ or $\mathrm{AgK} \alpha$ radiation as is common practice in high-pressure $\mathrm{x}$-ray studies. The restricted exit and entrance windows to the sample in the tetrahedral high-pressure apparatus ${ }^{5}$ used in this study severely reduce intensities. The statistical fluctuations due to low intensity further complicate the background elimination.

For reasons discussed later, the technique outlined in this paper restricts one to observing liquids at points near the melting curve. One is also limited to studying substances (a) the melting point of which never exceeds $700^{\circ} \mathrm{C}$, since the boron-plastic mixture used as a pressure-transmitting medium becomes unstable at high temperatures, and (b) which have low absorption coefficients suitable for an x-ray transmission method.

\section{APPARATUS}

This investigation was performed using the tetrahedral high-pressure diffractometer designed by Barnett and Hall ${ }^{5}$ at Brigham Young University. A commercial fine-focus ( $0.4 \mathrm{~mm}$ width) Mo target $\mathrm{x}$-ray tube was used to improve resolution.

Ordinarily, the use of a monochromator would further reduce the already low intensity associated with the high-pressure system. In this experiment however, a rather unique application of a commercially available doubly bent LiF monochromator provided a monochromatic beam without significant loss in $x$-ray intensities. Judicious placement of the monochromator made possible an increase in the $x$-ray intensity passing through the entrance and exit windows while maintaining resolution comparable to that which would be possible if only a singly bent crystal were used. 

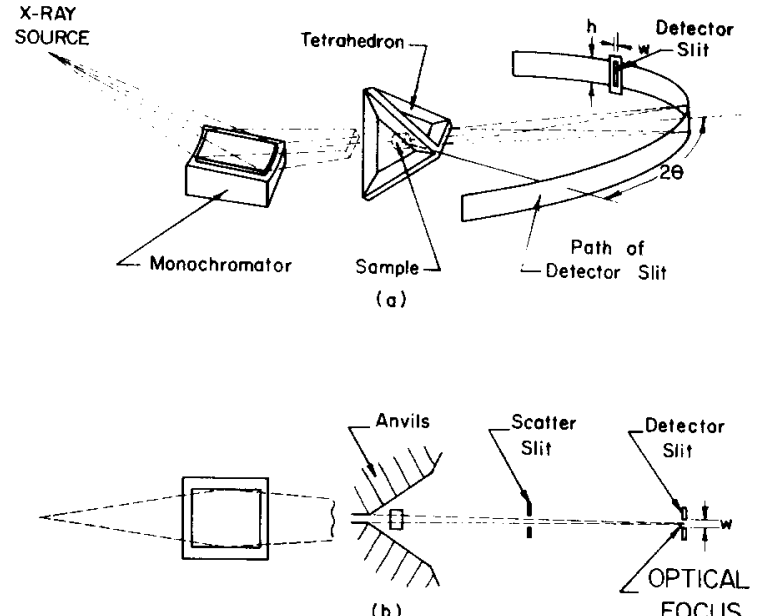

(b)

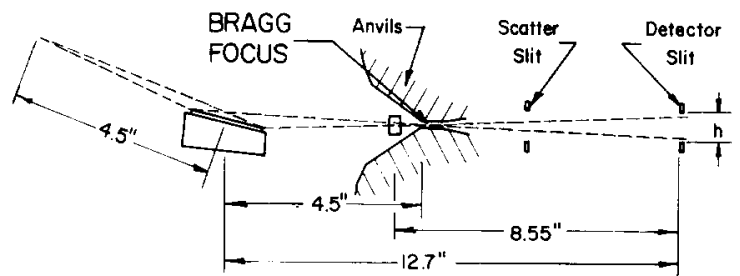

(c)

FIG. 1. Focusing properties of the doubly bent monochromator as it relates to the geometry of the tetrahedral press indicating the Bragg focus at the exit gasket and the optical focus at the detector slit: (a) three dimensional perspective view, (b) projection in the plane perpendicular to the $2 \theta$ detector scan, and (c) projection in the plane of the $2 \theta$ detector scan.

The doubly bent crystal was designed so that the Bragg focus occurs at a point 4.5 in. from its axis, and the optical focus occurs at $16 \mathrm{in}$. from its axis as illustrated in Fig. 1(a). Standard uses of this and other bent-crystal monochromators have been discussed extensively elsewhere..$^{6-8}$

Figures 1(b) and 1(c) show the focusing geometry of the monochromator as it relates to the pressure system. The crystal is placed 4.5 in. from the $x$-ray target and $4.5 \mathrm{in}$. from the exit gasket of the sample. The Bragg focal line at $4.5 \mathrm{in}$. is parallel to the exit gasket, and the optical focal line is perpendicular to the $2 \theta$ motion of the detector. This arrangement allows the maximum possible $\mathrm{x}$-ray intensity to pass through the sample and should provide good resolution by focusing. However, the poor quality of the optical focus for the commercially available monochromator crystals limits the resolution. Photographic determination of the focusing properties of the monochromator indicated no significant change in the width of the focal line within several inches of the specified optical focus. The original geometry of the tetrahedral x-ray press provided a distance of $\mathbf{1 2 . 7} \mathrm{in}$. from the monochromator to the detector slit as indicated in Fig. 1(b).
Since the optical focus at this distance was as good as that at the 16 in. distance specified by the manufacturer, no change in press geometry was required. The quality of the focus was indicated by a scan of the undeviated beam, which measured approximately 0.7 deg in width and was slightly asymmetrical. Since liquid patterns exhibit peaks which are greater than $2 \mathrm{deg}$ wide, this resolution is adequate.

A single-channel pulse-height selector was used to electronically discriminate against incoherent scattered radiation, fluorescent radiation, and wavelengths reflecting from the monochromator in second order. A front or scatter slit was placed on each counter as described by Barnett and Hall ${ }^{5}$ to discriminate against extraneous radiation scattered from the boron-plastic tetrahedron.

The method of sample construction is essentially that used by Barnett and Hall. ${ }^{5}$ An example of a typical sample design is that used for sodium and shown in Fig. 2. More details on this particular type of construction are given in Paper II of this series. Many features of this design will be common to any liquid study in the tetrahedral press. For example, all samples will require a heater, a thermocouple, a cell to contain the sample when molten, and a pressure-transmitting tetrahedron which is transparent to $\mathrm{x}$ rays. ${ }^{5,9}$ Care should be taken to exclude as much nonsample material as possible from the $x$-ray beam in order to minimize the extraneous contributions to the $x$-ray intensity.

\section{OBTAINING LIQUID COHERENT INTENSITY CURVES}

Elimination of the background intensity (the intensity scattered by the pressure-transmitting medium, cell, etc.) is one of the major problems in determining corrected $x$-ray intensities at these pressures. This elimination is accomplished by a subtraction process as follows. X-ray diffraction patterns of the sample in the solid state and in the liquid state near the melting curve are recorded. These patterns will be referred to hereafter as the solid and liquid patterns, respectively. The scattered intensity of each of the observed liquid and solid patterns may be written

$$
I^{\mathrm{obs}}=I^{\mathrm{coh}}+I^{\mathrm{inc}}+I^{\mathrm{back}}
$$

where we have separated the observed intensity into coherent and incoherent scattering from the sample and a background component which represents the contribution to the observed intensity by all of the other material in the beam such as the tetrahedron material, sample cell, etc.

If the solid pattern intensity is subtracted from the liquid-pattern intensity at each $2 \theta$ value, one obtains

$$
\begin{aligned}
& I_{\mathrm{liq}}{ }^{\text {obs }}-I_{\mathrm{sol}}{ }^{\text {obs }}=\left(I_{\mathrm{liq}}{ }^{\mathrm{coh}}-I_{\mathrm{sol}}{ }^{\mathrm{obh}}\right) \\
& +\left(I_{\mathrm{liq}}{ }^{\text {inc }}-I_{\mathrm{sol}}{ }^{\text {inc }}\right)+\left(I_{1 \mathrm{iq}}{ }^{\text {back }}-I_{\mathrm{sol}}{ }^{\text {back }}\right) .
\end{aligned}
$$

In order to obtain the radial distribution function it is 
necessary to obtain experimental values for $I_{1 \mathrm{iq}}{ }^{\mathrm{cob}}$ which have been corrected for polarization, absorption, etc. With appropriate simplifying assumptions all of the other terms on the right-hand side of Eq. (2) can either be eliminated or calculated as discussed below. The $x$-ray source is current-stabilized such that the intensity is constant and reproducible to less than $1 \%$; hence, one can assume that the incident beam intensity is the same for both patterns.

We have arbitrarily divided the coherent scattering from the solid $I_{\text {sol }}{ }^{\text {coh }}$ into two components: the intensity scattered into the characteristic peaks, $I_{\mathrm{sol}}{ }^{\mathrm{Bragg}}$, and the thermal diffuse scattering denoted by $I_{\mathrm{sol}}{ }^{\mathrm{TDS}}$. Analysis of thermal diffuse scattering from crystalline materials yields functions peaked at the Bragg positions. Our approach in separating $I_{\mathrm{sol}} \mathrm{Brags}^{\mathrm{Br}}$ and $I_{\mathrm{sol}}{ }^{\mathrm{TDS}}$ in effect assumes the use of the Debye theory involving independent vibration of the atoms ${ }^{10}$ and yields a relatively smooth function for the thermal diffuse scattering. Using this approximation, one can easily calculate the thermal diffuse scattering per atom. Evaluation of $I_{\mathrm{sol}}{ }^{\mathrm{TDS}}$, as well as the analysis of $I_{\mathrm{liq}}{ }^{\mathrm{coh}}$, requires a knowledge of the effective number of scattering atoms in the beam. This information can be determined only in conjunction with the normalization procedure presented later. The term which we will refer to as Bragg scattering, $I_{\mathrm{sol}}{ }^{\mathrm{Bragg}}$, is manifest in sharp peaks and this can be eliminated by the following experimental method. Most sample materials tend to form large single crystals near the melting temperature. When monochromatic radiation is incident on a single crystal, no Bragg peaks will be observed unless the crystal is oriented at the proper angle to the primary beam given by Bragg's law. It is unlikely that any of the crystals will be oriented relative to the primary beam and also to the press in such a fashion that $x$ rays of the wavelength used will be Bragg-diffracted out through a gasket to the counters. If such single-crystal diffraction is observed, it is easily identifiable. The sample may then be melted and resolidified repeatedly, as detected by the presence or absence of the broad liquid peak, until no single-crystal peaks are observed for the sample. This condition seldom required more than two trial meltings in the case of sodium if the solidification was accomplished slowly. This procedure provides a means of experimentally reducing $I_{\mathrm{sol}}{ }^{\mathrm{Bragg}}$ in Eq. (2) to zero for that pattern, and the recorded solid pattern contains only background (including background peaks), incoherent scattering, and thermal diffuse scattering from the solid sample. It is possible that a small sample crystal would diffract with an intensity so small as not to be visually observed but yet large enough to become significant after the $I_{1 \mathrm{iq}}{ }^{\text {obs }}-I_{\text {sol }}{ }^{\text {obs }}$ subtraction is made. This possibility represents one of the serious unresolved experimental problems of the study.

The incoherent scattering intensity for many simple materials has been calculated on a theoretical basis (for example, see Freeman ${ }^{11}$ ). The incoherent scatter-

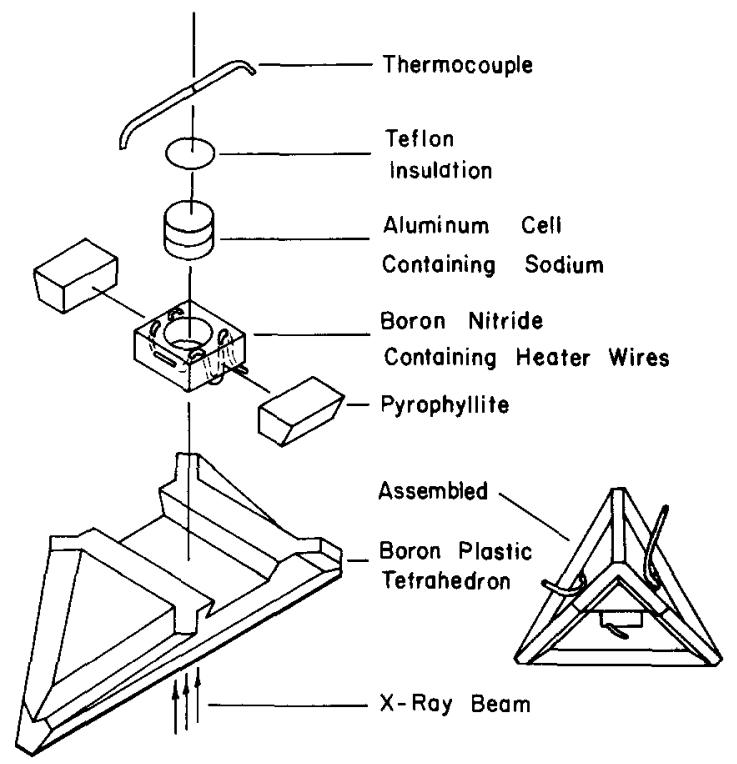

FIg. 2. Sample design used for $\mathrm{Na}$ showing an expanded view and an assembled tetrahedron.' The top half of the tetrahedron has been omitted for simplicity in the expanded view.

ing intensity is directly proportional to the number of atoms in the incident beam. If we assume that the average incoherent scattering per atom is independent of the state of the substance, the term in Eq. (2) for the difference in incoherent scattering intensity from the liquid and the solid becomes proportional to the difference in the number of atoms in the incident beam. Simple calculations involving the density change across melting show that in most cases, and in particular for sodium, the difference in incoherent intensities is less than $2 \%$ of the incoherent intensity scattered by the liquid, which intensity is already small compared to the coherent intensity at most angles. The incoherent intensity difference term in Eq. (2) can thus be neglected.

The principal contribution to the background radiation is the scattering from the tetrahedron and from the sample cell materials. Since these materials are not involved in the meiting transformation, their diffracted intensities should change only due to temperature variation. If the solid and liquid patterns are taken at temperatures near the melting curve, the difference $I_{\mathrm{liq}}{ }^{\text {back }}-I_{\text {sol }}{ }^{\text {back }}$ should be small and unstructured even though large peaks exist in the background radiation. When this difference term is neglected, Eq. (2) becomes

$$
I_{1 \mathrm{iq}}{ }^{\text {obs }}-I_{\mathrm{sol}}{ }^{\text {obs }}=I_{1 \mathrm{iq}}{ }^{\text {ohh }}-I_{\mathrm{sol}}{ }^{\mathrm{TDS}} \text {. }
$$

Typical solid and liquid patterns as obtained for sodium are shown as an example in Figs. 3(a) and 3 (b). When they are subtracted, the difference curve shown in Fig. 3(c) is obtained. As can be observed, the difference pattern defines very well the features of 


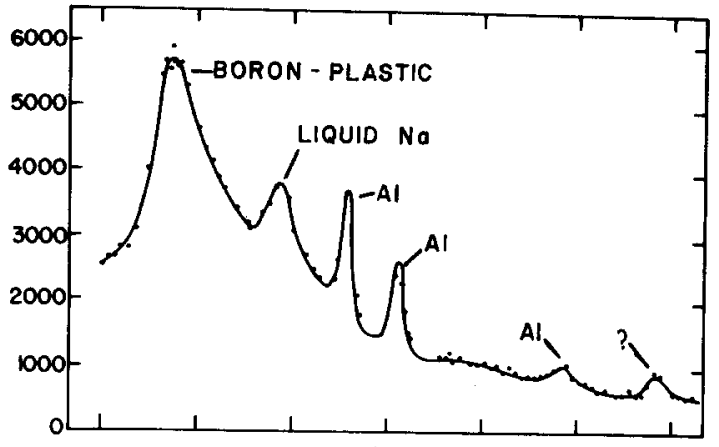

(a)

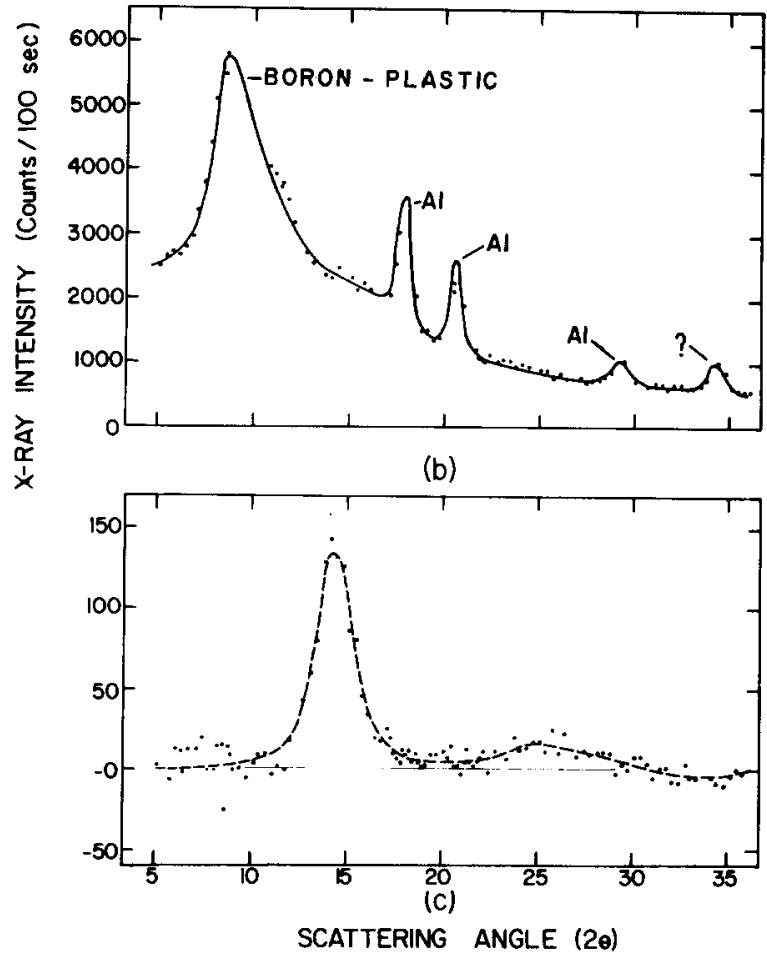

FIG. 3. Typical patterns obtained in the case of $\mathrm{Na}$ at $17 \mathrm{kbar}$ : (a) a liquid pattern with extraneous background peaks and the first liquid $\mathrm{Na}$ peak indicated, (b) the solid pattern associated with (a) with extraneous background peaks indicated and (c) the difference pattern obtained by subtracting, point by point, the solid pattern (b) from the liquid pattern (a).

the dominant peak but is far from ideal in the largeangle region where the less pronounced features of the pattern exist. Such results are not unexpected when statistical scatter and problems such as small, undetected Bragg peaks are considered. We know that an ideal coherent $\mathrm{x}$-ray pattern from a liquid is smooth with no sharp variations beyond the dominant peak. Since the term $I_{\mathrm{sol}}{ }^{\text {TDS }}$, calculated using the independentatom approximation mentioned earlier, is also an unstructured function, the difference pattern should also be relatively smooth. The only structure expected should be two or three broad peaks. In Fig. 3(c) only one of these liquid peaks is clearly discernible. The ordinate in Figs. 3(a) and 3(b) represents the number of counts recorded during 100 -sec counting intervals.
Each solid and liquid pattern required approximately $6 \mathrm{~h}$ scanning time. The average difference in the solid and liquid patterns at large angles was often of the order of the statistical variation in the recorded counts in each pattern as seen in Figs. 3(a)-3(c). To reduce statistical fluctuations and randomize errors associated with the subtraction process, we obtained 8-10 difference patterns at each pressure studied. Those regions in each pattern where there were sudden changes in intensity were eliminated and replaced by a smooth curve. The patterns were then combined and a smooth curve drawn through the composite points. Details of this process are discussed in the dissertation associated with this work. ${ }^{12}$ The resulting intensity pattern will be referred to hereafter as the composite pattern, $I^{\mathrm{comp}}$. The composite pattern associated with the curves Fig. $3(\mathrm{a})-3$ (c) is shown in Fig. 4. All patterns combined to make the composite curve must be at the same pressure and temperature. Since pressures in the tetrahedral press cannot be precisely reproduced, the most consistent results are obtained when a single sample is raised to a given pressure and temperature and 8-10 pairs of solid and liquid patterns are obtained without changing the pressure or the temperature.

\section{CORRECTIONS AND REFINEMENT OF COHERENT LIQUID INTENSITY}

In the previous section we showed how one can obtain the composite curve of $I_{\mathrm{liq}}{ }^{\mathrm{coh}}-I_{\mathrm{sol}}{ }^{\mathrm{TDS}}$ from the observed intensity patterns. These smoothed experimental curves now need to be corrected for known instrumental effects, adjusted by extrapolation, and then normalized to electron units before the RDF can be calculated.

The absorption correction is complicated by pressureinduced changes in (1) the geometrical relationships inside the tetrahedron as the sample cell collapses and

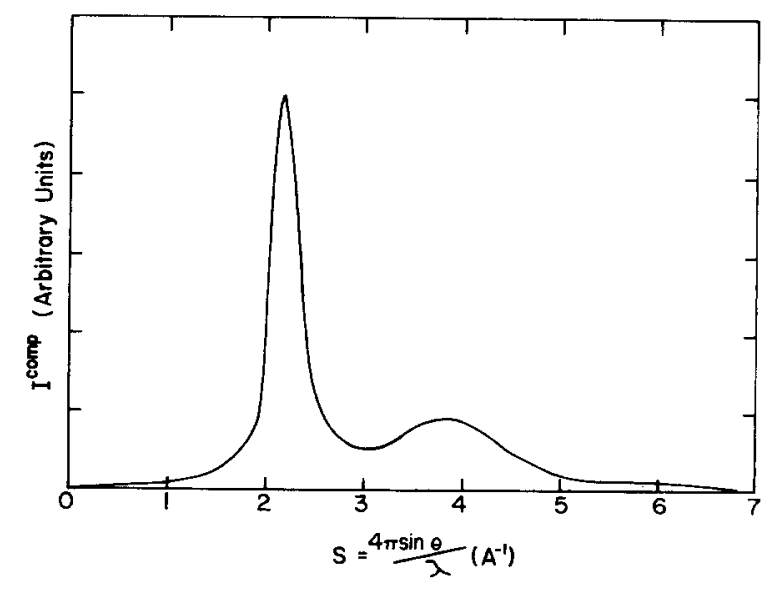

Fig. 4. Composite intensity, $I^{\mathrm{comp}}=I_{\mathrm{liq}}{ }^{\mathrm{coh}}-I_{\mathrm{Bo}}{ }^{\mathrm{TDS}}$, for liquid $\mathrm{Na}$ at $17 \mathrm{kbar}$ after extrapolation to zero $S$ and to large $S$. 
changes shape and as the gaskets are extruded, and (2) the density of materials and, hence, the linear absorption coefficient. When the sample is initially placed in the press, the absorption correction is straightforward because the geometry consists of a series of planes perpendicular to the incident beam. In the absence of any information about the shape of the collapsed cell at high pressures, we assumed that the geometry remains planar. By using the compressibility data available on the materials involved, the change of the linear absorption coefficient with pressure can be readily calculated. By making careful measurements of the sample before and after the experiment and by assuming that most of the flow in the gasket region takes place at low pressures, an appropriate absorption factor can be calculated. It is estimated that the maximum observed error in the intensity pattern due to the absorption correction is probably less than $10 \%$. The error should be much less than this at the smaller angles where the principal peaks occur and will have negligible effect on the position of those peaks.

The corrections for polarization and fluorescence are straightforward. The polarization factor used ${ }^{13}$ is for an ideally mosaic LiF monochromator crystal. Fluorescence should be negligible in this experiment since the wavelength of the MoKa radiation used is far from the $K$ and $L$ absorption edges in sodium.

Each individual difference pattern, such as the one in Fig. 3(c), is poorly defined for scattering angles less than about $10 \mathrm{deg}$ and greater than about $30 \mathrm{deg}$. The composite curve in Fig. 4 has been extrapolated to theoretical values at small angles and at large angles as is required to calculate the RDF. The extrapolation at small angles is discussed by Mikolaj and Pings ${ }^{14}$ and has negligible effect on the resulting RDF. At large angles $I_{\mathrm{liq}}{ }^{\mathrm{coh}}-I_{\mathrm{sol}}{ }^{\mathrm{TDS}}$ is extrapolated to oscillate about zero since both $I_{\mathrm{liq}}{ }^{\text {coh }}$ and $I_{\text {sol }}{ }^{\text {TDS }}$ theoretically approach the square of the atomic scattering factor as the diffraction angle increases. A logical means of refining this wide-angle extrapolation will be presented later.

\section{CALCULATION OF THE RADIAL DISTRIBUTION FUNCTION}

The radial distribution function, $\mathrm{RDF}=4 \pi r^{2} \rho(r)$, is obtained from the coherent intensity curve using the procedure summarized by Warren. ${ }^{10}$ Since the intensity curves are in arbitrary units, one must convert to electron units by dividing each composite curve by an appropriate factor $N$, which represents the effective number of scattering atoms. The standard treatment relies upon the assumption that at large angles the coherent intensity scattered from the liquid should approach the scattering from independent atoms, the atomic scattering function $f^{2}$. The factor $N$ cannot be experimentally measured and is selected to make the observed coherent intensity meet this criterion.

To emphasize structural effects a new intensity function $i(S)=\left(I_{\mathrm{liq}}{ }^{\text {coh }} / N-f^{2}\right) f^{-2}$ is usually defined where
$S=4 \pi \sin \theta / \lambda$. The function $i(S)$ represents the fractional difference per atom between the coherent intensity scattered by the liquid and that scattered by independent atoms. The fundamental equation ordinarily used to calculate the RDF is

$$
4 \pi r^{2} \rho(r)=4 \pi r^{2} \rho_{0}+\left(\frac{2 r}{\pi}\right) \int_{0}^{\infty} S i(S) \sin r S d S,
$$

where $\rho_{0}$ is the average atomic density.

The integration in Eq. (4) should extend from zero to infinity. Since the observed intensities were obtainable only over a range of scattering angle, $2 \theta$, from 10 to $30 \mathrm{deg}$ (corresponding to a range of $S$ of about 1.75 to $5.5 \AA^{-1}$ ), it is necessary to extrapolate as mentioned above. There is no need to extrapolate to infinity since the integrand becomes zero for large values of $S$. Evaluation of the integral with some upper limit $S_{m}$ introduces certain termination errors into the RDF such as false peaks and ripple at small $r$ values. These effects are discussed in detail elsewhere. ${ }^{15,16}$ The larger the value of $S_{m}$, the smaller these termination errors will be. However, if the intensity is extrapolated incorrectly to large $S$ values, extrapolation errors in the RDF will result. Errors in the initial extrapolation become obvious after $i(S)$ has been obtained since it is known that $i(S)$ must oscillate about and approach zero as $S$ increases. An improved extrapolation can be made at this stage of the analysis.

The method used in this experiment to calculate the normalization factor $N$ is a modification of the method developed by Krogh-Moe ${ }^{17}$ and Norman. ${ }^{18}$ Generally one requires

$$
N=\int_{0}^{S_{m}} I_{1 \mathrm{iq}}{ }^{\mathrm{coh}} S^{2} d S / \int_{0}^{S_{m}} f^{2} S^{2} d S
$$

which determines the factor $N$ such that $I_{\mathrm{liq}}{ }^{\mathrm{en}} / N$ approaches $f^{2}$ at large angles. To achieve this condition in the present study $I^{\mathrm{comp}} / N=\left(I_{\mathrm{liq}}{ }^{\text {chh }} / N\right)-\left(I_{\mathrm{sol}}{ }^{\mathrm{TDS}} / N\right)$ must approach $f^{2}-I_{\mathrm{sol}}{ }^{\mathrm{TDS}} / N$ at large angles. Theoretically the thermal diffuse scattering per atom, $I_{\text {sol }}{ }^{\text {TDS }} / N$, is known. ${ }^{10}$ The expression used for $N$, which takes the thermal diffuse term into account, thus becomes

$$
N=\int_{0}^{s_{m}} I^{\mathrm{comp}} S^{2} d S /\left(\int_{0}^{s_{m}} f^{2} S^{2} d S-\int_{0}^{s_{m}} \frac{I_{\mathrm{sol}}^{\mathrm{TDS}}}{N} S^{2} d S\right)
$$

Once $N$ has been determined, the thermal diffuse scattering per atom can be added to $I^{\mathrm{comp}} / N=I_{\mathrm{liq}}{ }^{\text {coh }} / N-$ $I_{\text {sol }}{ }^{\text {TDS }} / N$ to yield the coherent intensity scattered from the liquid in electron units, e.u. The coherent intensity curve obtained in this manner does, in fact, approach the $f^{2}$ curve as desired. It should be noted that the determination of $N$ from the composite patterns in this manner increases the sensitivity of $N$ to small errors in any of the intensity functions $I^{\text {omp }}, f^{2}$, or $I_{\mathrm{sol}} \mathrm{TDS} / N$. Detailed analysis indicates that this sensi- 


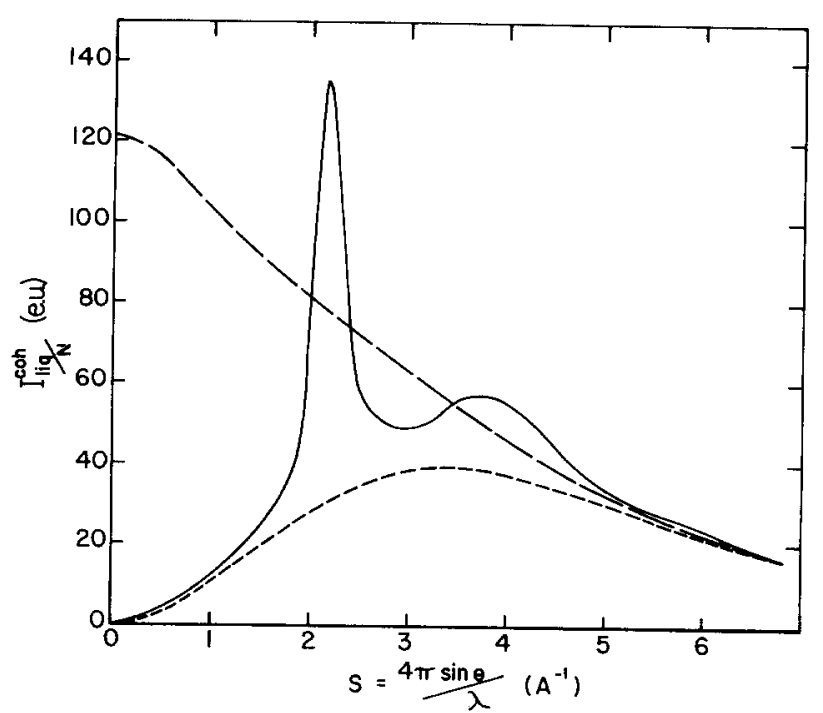

FIG. 5. Normalized coherent $x$-ray intensity $I_{1 \mathrm{iq}}{ }^{\mathrm{eoh}} / N$ for liquid $\mathrm{Na},-$, compared to the atomic scattering factor $f^{2},--$ and the thermal diffuse scattering per atom $I_{\text {sol }}$ TD8 $/ N$ from solid $\mathrm{Na}, \ldots$, all at 17 kbar.

tivity seriously affects the amplitude but not the peak positions in $I_{1 \mathrm{iq}}{ }^{\text {coh }} / N, i(S)$, and the RDF.

The thermal diffuse scattering per atom has been calculated on a theoretical basis for both single crystals $^{10}$ and for powdered solids ${ }^{19,20}$ in the cubic structure. These calculations yield peaked functions. Since the intensity patterns have been smoothed as discussed above, we have used the approximation of independent atomic vibrations proposed by Debye for the thermal diffuse scattering. Thus, $I_{\mathrm{sol}}{ }^{\mathrm{TDS}} / N$ is the unstructured function $I_{\text {sol }}{ }^{\mathrm{TDS}} / N=f^{2}\left(1-e^{-2 M}\right)$ where $M$ is the commonly defined quantity

$$
M=6 h^{2} / m k \theta[\phi(x) / x+1 / 4](\sin \theta / \lambda)^{2} .
$$

As an example, Fig. 5 shows the $I_{\text {liq }}{ }^{\text {coh }} / N$ curve which was obtained from the $I^{\text {comp }}$ in Fig. 4 after adding $I_{\text {sol }}{ }^{\mathrm{TDS}} / N$. The thermal diffuse scattering and the atomic scattering intensity curves are also shown for comparison purposes.

\section{DISCUSSION}

The two quantities $\rho_{0}$ and $f$ must be independently known at each pressure before the RDF can be determined from the $x$-ray intensity data. The average density of the liquid, $\rho_{0}$, is only slightly different from the density of the solid near the melting temperature at pressure. The density of the solid is known or can be obtained for many materials, and one can estimate the difference by using the melting curve and the Clausius-Clapeyron equation and assuming the change in entropy due to melting is approximately constant along the melting curve.

The calculated values of $f$ in the standard tables apply to isolated atoms or ions at atmospheric pressures.
Since errors in $f$ will be reflected in the RDF, we must consider the effect of pressure and of the state of the material on $f$. In the case of sodium, the atomic scattering factor has been calculated by $\mathrm{Cromer}^{21}$ over the range of pressures involved with this study and has been shown to vary by less than one percent. Sodium is a relatively compressible material, and one would expect less compressible materials to have even less variation in the atomic scattering factor. Estimates of the difference in $f$ produced by a change in the state of the material are less than the difference between the various theoretical calculations ${ }^{22}$ and represent an error common to all diffraction studies.

The radial distribution function for $\mathrm{Na}$ at $17 \mathrm{kbar}$, calculated from the coherent intensity curve in Fig. 5, is shown in Fig. 6. The ripple at small $r$ values is an indication of the errors introduced in the RDF by errors in the absorption correction, normalization, intensity measurements, $f^{2}$, and by termination errors, extrapolation errors, etc. The observed ripple is 2 to 3 times as high as is often reported at atmospheric pressure. The ripple has been very similar in all the radial distribution functions determined in this study and indicates a systematic effect for which we are unable to account. A number of attempts have been made to reduce the magnitude of the ripple. Since $i(S)$ involves the coherent intensity divided by $f^{2}$, which

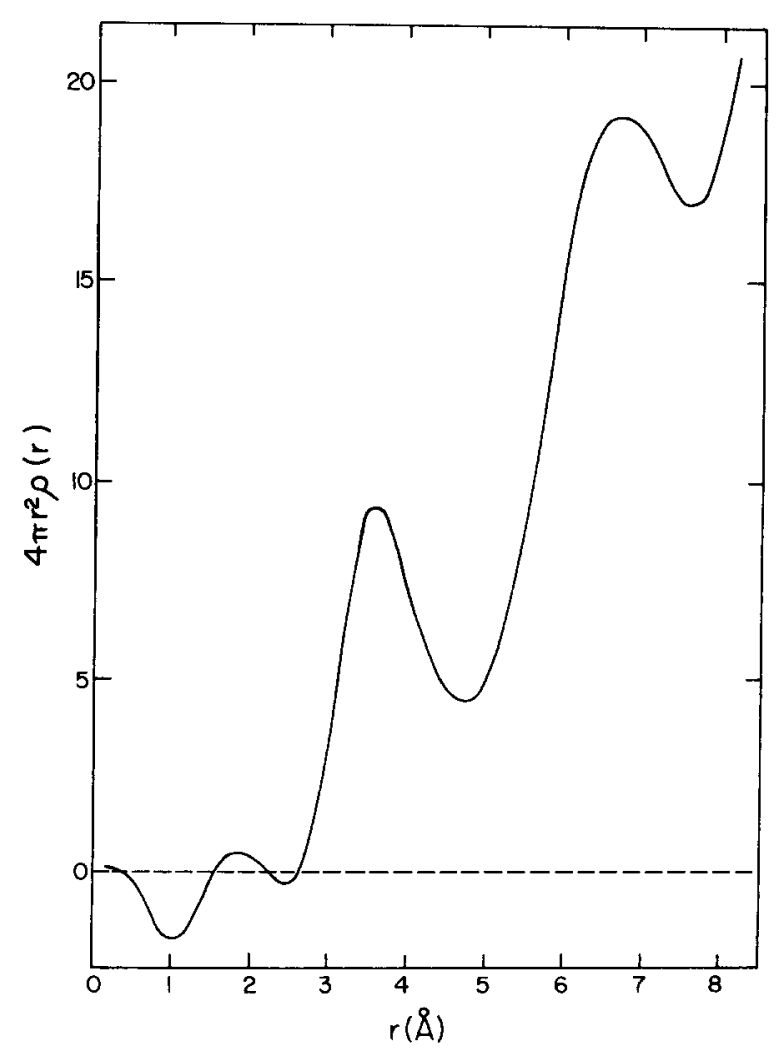

FIG. 6. Radial distribution function, $\mathrm{RDF}=4 \pi r^{2} \rho(r)$, for liquid $\mathrm{Na}$ at 17 kbar. 
itself is small at large angles, errors in the coherent intensity at these large angles are enhanced. Furthermore, since many of the corrections which have been made tend to be a maximum at large angles where the intensities are least known, significant errors may arise in the calculation of the RDF. The method often used to minimize this problem is to multiply $i(S)$ by an artificial temperature factor, $\exp \left(-A \sin ^{2} \theta\right)$, thus diminishing the importance of the wide-angle values. However, the artificial temperature factor decreases resolution in the RDF, hence lowering the detectability of small ordering or structural changes in the liquid. The use of the artificial temperature factor and its effect on the RDF are summarized elsewhere. ${ }^{15,23}$ The use of this factor did reduce the ripple in the RDF, indicating the existence of errors in the wide-angle region. The normalization constant $N$ was varied without success in reducing the ripple, which fact indicates that the method already discussed of calculating $N$ yields reasonable values.

\section{CONCLUSIONS}

In evaluating the technique of this paper it should be remembered that the technique is concerned primarily with changes in the intensity pattern and in the radial distribution function as the pressure is changed rather than with absolute measurements. Thus, systematic effects which are not pressure sensitive should not influence determination of the pressureinduced variations being studied. If all sets of data are analyzed in the same manner, errors resulting from faulty estimates of true corrections will be diminished or entirely eliminated unless the errors are pressure dependent. In order to estimate the importance of errors in the correction terms as they affect the final calculations of the radial distribution function, we assumed values for the correction terms that were intentionally in error by an amount in excess of any anticipated. The resulting changes in the RDF established effective error flags for values reported in Paper II of this series and gave indication that the errors introduced by inaccurate and uncertain approximations were reasonable.

The degree of success achieved in $\mathrm{x}$-ray diffraction of liquids at high pressures is illustrated by the data which have been observed and analyzed for liquid sodium. These data will be reported in detail and the reliability of the technique will be evaluated in Paper II of this series. For the present, note that the procedure of subtracting a solid pattern from a liquid pattern to eliminate background, incoherent scattering, and fluorescent radiation and the procedure of adding the thermal diffuse scattering associated with the solid back into the intensity patterns appear to yield interpretable, coherent intensity patterns. The changes in the dominant features of the intensity pattern and the $\mathrm{RDF}$ as pressure is increased along the melting curve are clearly experimentally observable using the techniques explained herein.

\footnotetext{
* This work was supported in part by a grant from the National Science Foundation, and the study formed the basis for the doctoral dissertation of $\mathbf{K}$. $\mathrm{H}$. Brown (Brigham Young University, 1969), who was supported by a NASA fellowship during part of the study.

$\dagger$ Present address: Department of Physics and Earth Sciences, California State Polytechnic College, Pomona, Calif.

1 K. Furukawa, Rept. Progr. Phys. 25, 395 (1962).

2 N. S. Gingrich, Rev. Mod. Phys. 15, 90 (1943).

${ }^{3}$ R. F. Kruh, Chem. Rev. 62, 319 (1962).

${ }^{4} \mathrm{~K}$. H. Brown and J. D. Barnett, J. Chem. Phys. 57, 2016 (1972), following paper.

${ }^{B}$ J. D. Barnett and H. T. Hall, Rev. Sci. Instr. 35, 175 (1964).

${ }^{6}$ L. S. Birks and R. T. Seal, J. Appl. Phys. 28, 541 (1957).

7 D. R. Chipman, Rev. Sci. Instr. 27, 164 (1956).

${ }^{8}$ H. J. Garrett and H. A. Lipsitt, Rev. Sci. Instr. 32, 942 (1961).

${ }^{\circ} \mathrm{V}$. E. Bean, master's thesis, Brigham Young University, 1964.

${ }^{10}$ B. E. Warren, $X$-ray Diffraction (Addison-Wesley, Reading, Mass., 1969).

${ }^{11}$ A. J. Freeman, Acta Cryst. 13, 190 (1960).

$12 \mathrm{~K}$. H. Brown, Ph.D. dissertation, Brigham Young University, 1969.

${ }^{13}$ S. Miyake, S. Togawa, and S. Hosoya, Acta Cryst. 17, 1083 (1964).

${ }_{14}$ P. G. Mikolaj and C. J. Pings, J. Chem. Phys. 46, 1401 (1967).

${ }_{15} \mathrm{~J}$. Waser and V. Schomaker, Rev. Mod. Phys. 25, 671 (1953).

${ }_{16} \mathrm{D}$. Stirpe and C. W. Tompson, J. Chem. Phys. 36, 392 (1962).

${ }_{17}$ J. Krogh-Moe, Acta Cryst. 9, 951 (1956).

18 N. Norman, Acta Cryst. 10, 370 (1957).

${ }^{19} \mathrm{~F}$. H. Herbstein and B. L. Averbach, Acta Cryst. 8, 843 (1955).

${ }^{20}$ B. E. Warren, Acta Cryst. 6, 803 (1953).

21 D. T. Cromer (private communication, 1970).

${ }^{22}$ H. Ocken and C. N. J. Wagner, Phys. Rev. 149, 122 (1966).

${ }^{23} \mathrm{~W}$. L. Bragg and J. West, Phil. Mag. 10, 823 (1930).
} 